\title{
Contours Matching with A Text-based Method
}

\author{
Saliha Aouat \\ LRIA Laboratory, Computer Science Department, USTHB University, Algiers, Algeria \\ E-mail: saouat@usthb.dz \\ Slimane Larabi \\ LRIA Laboratory, Computer Science Department, USTHB University, Algiers, Algeria \\ E-mail: slarabi@usthb.dz
}

\begin{abstract}
We propose in this paper a method to match silhouettes. Silhouettes are described with a textbased representation. An iterative process is used to reduce descriptors. When the size of a little part is negligible in relation with sizes of main parts, that little part will be considered as noisy and will be suppressed from the initial textual descriptor. After the reduction process, the descriptors can be compared in order to perform the matching process.
\end{abstract}

Index Terms - Descriptors, Matching, Reduction, Textual Descriptors, Noisy Parts

\section{Introduction}

Intensity-based and Geometric-based methods are two general and most popular approaches for image matching. Intensity-based (color and texture), work with the intensity of the pixels and use the image itself as a feature descriptor. Geometry-based however, deal with the shape; they are feature-based method that extract points from the image (usually edge or corner points) and reduce the problem to point set matching. Users are more interested in matching and retrieval by shape than by color and texture ${ }^{[1][2][3][4]}$.

Geometric methods are adapted to assumptions made about the illumination and viewpoint variations existing between two images. They determine the optimal matching of two patch-based image representation under rotation, scaling, and translations. Typically objects are subject to quite complex transformations when projected into an image plane. Therefore, the real transformations are approximated by easier transformations that can be mathematically treated with reasonable effort.

Different representations of shapes have been proposed these last years and used in the recognition process. The most known representations are based onto appearance ${ }^{[1]}$, outline contour [2][3][4][5][6][7][8], aspect-graph ${ }^{[9][10]}$, set of characteristic outline points ${ }^{[11]}$, medial axis of silhouettes ${ }^{[12][13]}$, shock graph ${ }^{[14][15]}$, and shape axis trees (S-A-trees) ${ }^{[16]}$. A review of shape representation methods may be found in ${ }^{[17][18]}$. In ${ }^{[19]}$, authors propose a part-based method for silhouettes representation. Silhouettes are partitioned into parts, junction and disjunction lines. Each element is then geometrically described. The obtained description is written following an XML language noted XLWDOS (XML Language for Writing Descriptors of Outline Shapes). Since real images are noisy, there XLWDOS descriptors may be very different. This sensitiveness to noise does not facilitate their use in the matching and recognition processes.

A notion of multi-scale descriptors of silhouettes is introduced to match silhouettes ${ }^{[20]}$. A Gaussian convolution of silhouettes is applied in order to smooth outline shapes and eliminate noise depending on the value of the Gaussian scale. Also, noisy XLWDOS descriptors of silhouettes may be matched using a reduction technique that eliminates noisy elements from the descriptors.

This paper is structured as follows:

We present in the second section an overview of the part-based method for describing outline shapes. In the third section we show the sensitiveness to noise of XLWDOS descriptors. In the fourth section, we explain our strategy based on the matching of XLWDOS descriptors using the reduction technique. The proposed method is validated using real images and the obtained results are presented and discussed in the fifth section.

\section{Silhouettes Description}

Concavity points for which direction of outer contour changes following top-bottom-top or bottomtop-bottom are considered as partition points (see Figure 1.a). A silhouette is partitioned at these points onto parts, junction and disjunction lines: either, two parts or more are joined with a third part through a junction line, or a part is joined with two parts or more through a disjunction line. This process applied to the left silhouette in Figure 1 produces seven parts, two junction lines and one disjunction line. The silhouette descriptor is the grouping of descriptors of its elements. A part is defined by its two boundaries (left and right) which begin at the highest left point and terminate at 
their lowest points (see Figure 1.b). Using the inflection points, these boundaries are segmented into a set of primitives (line, convex and concave contours) and described by the parameters: type (line, convex or concave curve), degree of concavity or convexity, angle of inclination and length. Junction and disjunction lines are decomposed onto segments. Each segment is described with three parameters: type, the reference numbers of parts where it appertains and its length. Types of segment are: Junction if it is common for two parts, Free-High if it belongs only to the high part or Free-Low if it belongs to the low part. Applying this description to write the descriptor of part $\mathrm{P}_{2}$ of the shape in the left of Figure 1, we obtain:

$P_{2} \rightarrow<P_{2}><L>C V \quad 6 \% 56 \quad 76</ L><R>C V \quad 16 \% 107$ $77</ R></ P_{2}>$

This descriptor is read as follow: The left boundary of Part 2 is composed by a convex contour with 0.06 as degree of convexity, $56^{\circ}$ of inclination and 76 pixels as length. The right boundary is composed by a convex contour with 0.16 as degree of convexity, $107^{\circ}$ of inclination and 77 pixels as length.

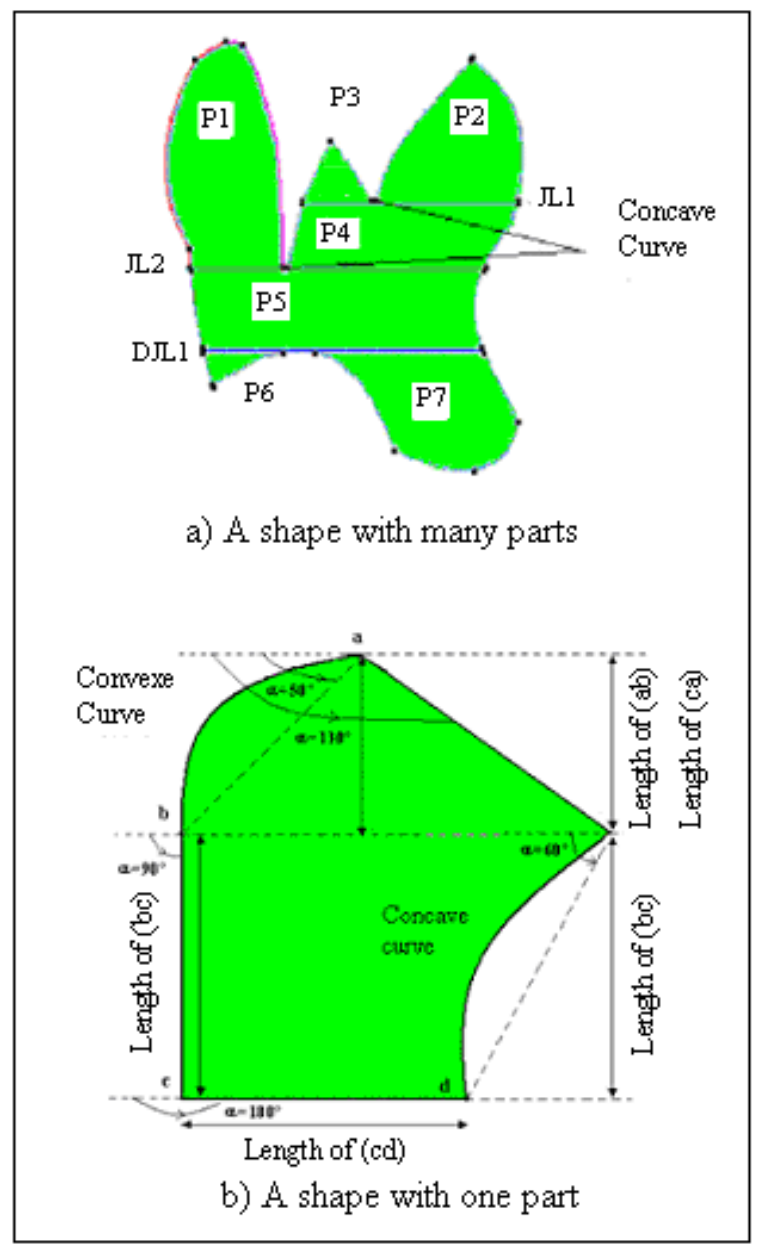

Fig. 1: Example of a silhouette, concave points, and parts
The notion of composed part is defined as a set of two (or more) parts joined to another part using a junction (or a disjunction) line and written as follows:

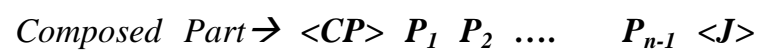
Junction line $</ \boldsymbol{J}>\boldsymbol{P}_{\boldsymbol{n}}</ \boldsymbol{C P}>\quad /<\boldsymbol{C P}>\boldsymbol{P}_{\boldsymbol{1}}<\boldsymbol{D}>$ Disjunction line $</ \boldsymbol{D}>\boldsymbol{P}_{2} \boldsymbol{P}_{3} \ldots . \boldsymbol{P}_{\boldsymbol{n}}</ C \boldsymbol{P}>$

Recursively, a composed part is considered as a part and may constitute (with other elements) other composed parts.

There are three composed parts in the XLWDOS descriptor of the left silhouette in Figure 1:

$$
\begin{aligned}
& \text { - } \leq C P>P_{2} \underline{P}_{3}<J>J L_{1}</ J>P_{4} \leq / C P> \\
& \text { - }\left\langle C P>P_{1}<C P>P_{2} P_{3}<J>J L_{1} \leq / J>P_{4}</ C P>\right. \\
& <J>J L_{2}</ J>P_{5}</ C P> \\
& \text { - } \quad\left\langle\mathrm{CP}>\quad\left\langle C P>\quad P_{1} \quad \leq C P>P_{2} \quad P_{3} \quad<J>J L_{1}</ J>\right.\right. \\
& \underline{P}_{4}</ C P><J>J L_{2}</ J>P_{5}</ C P><\mathrm{D}>\mathrm{DJL} 1</ \mathrm{D}>\mathrm{P}_{5}
\end{aligned}
$$

To write descriptors of silhouettes we use the following syntax:

Silhouette $\rightarrow<$ DXLWDOS $><$ Name $>$ Objectname $></$ Name $>$ Composed Part $</$ DXLWDOS $>$

(DXLWDOS means description according to XLWDOS description).

Finally, the descriptor of the silhouette in Figure 1 is:

$$
<\text { DXLWDOS }><\text { Name }>\text { Silhouette } 1><\text { /Name }>
$$

$<\boldsymbol{C P}>\left\langle\boldsymbol{C P}><\boldsymbol{P}_{1}\right\rangle<\boldsymbol{L}>$ r 3210 cv 14\% 88102 r 9010 $</ \mathbf{L}><\boldsymbol{R}>$ r $1658 \mathrm{cv} 6 \% 100120</ \boldsymbol{R}></ \boldsymbol{P}_{1}>$

$\begin{array}{llllll}<\boldsymbol{C}><\boldsymbol{P}_{2}><\boldsymbol{L}>\text { CV } & 6 \% & 56 & 76</ \boldsymbol{L}><\boldsymbol{R}>\text { CV } & 16 \% & 107\end{array}$ $77</ \boldsymbol{R}></ \boldsymbol{P}_{2}>$

$\left\langle\boldsymbol{P}_{3}><\boldsymbol{L}>\right.$ r $6432</ \boldsymbol{L}><\boldsymbol{R}>$ r $12332</ \boldsymbol{R}></ \boldsymbol{P}_{3}><\boldsymbol{J}>\mathbf{j}$ P3 P4 36 w P4 2 j P2 P4 75</J $>$

$\begin{array}{lllllll}\left\langle\boldsymbol{P}_{4}><\boldsymbol{L}>\right. & r & 76 & 34 & </ \boldsymbol{L}><\boldsymbol{R}> & r & 63\end{array}$ $34</ \boldsymbol{R}></ \boldsymbol{P}_{4}></ \boldsymbol{C P}><\boldsymbol{J}>h$ P1 1 j P1 P5 48 w P5 2 j P4 P5 103 h P4 $1</ J>$

$\begin{array}{llllll}\left\langle\boldsymbol{P}_{5}><\boldsymbol{L}>\right. & r & 98 & 41</ \boldsymbol{L}><\boldsymbol{R}>\text { CC } & 11 \% & 88\end{array}$ $41</ \boldsymbol{R}></ \boldsymbol{P}_{5}></ \boldsymbol{C P}><\boldsymbol{D}>$ j P5 P6 42 h P5 17 j P5 P7 87 w P7 $1</$ D $>$

$\left\langle\boldsymbol{P}_{6}><\boldsymbol{L}>\operatorname{rin} 105</ \mathbf{L}><\boldsymbol{R}>\right.$ CC 6\% $2719</ \boldsymbol{R}></ \boldsymbol{P}_{6}>$

$\left\langle\boldsymbol{P}_{7}><\boldsymbol{L}>\right.$ CC $10 \% 12753 \mathrm{cV} 7 \% 165 \quad 11</ \mathbf{L}><\boldsymbol{R}>r$ 11538 cv $17 \% 4826</ \boldsymbol{R}></ \boldsymbol{P}_{7}></ \boldsymbol{C P}>$

$</ D X L W D O S>$

\section{Sensitiveness to Noise of XLWDOS Descriptors}

XLWDOS descriptors are sensitive to noise which may produce additional parts, junction and disjunction lines. Noise may then change the global structure of XLWDOS descriptor, but in other cases it changes 
only the geometric description of its elements. Figure 2.b illustrates an example where noise produces in addition, two parts, one junction line, two other parts, and one disjunction line relatively to the shape 2.a. Also, noise may correspond to concave points that the change of position creates new parts and lines. The silhouette of Figure 2.d illustrates an example where a concavity point change of position produces additional parts and lines relatively to the shape 2.c.

Finally, the XLWDOS descriptors are robust to noise when it changes only the geometric description of contours; it is the case of the silhouette 2.e. Depending on the direction of computation of the XLWDOS descriptor; noise may change the global structure of XLWDOS descriptors.

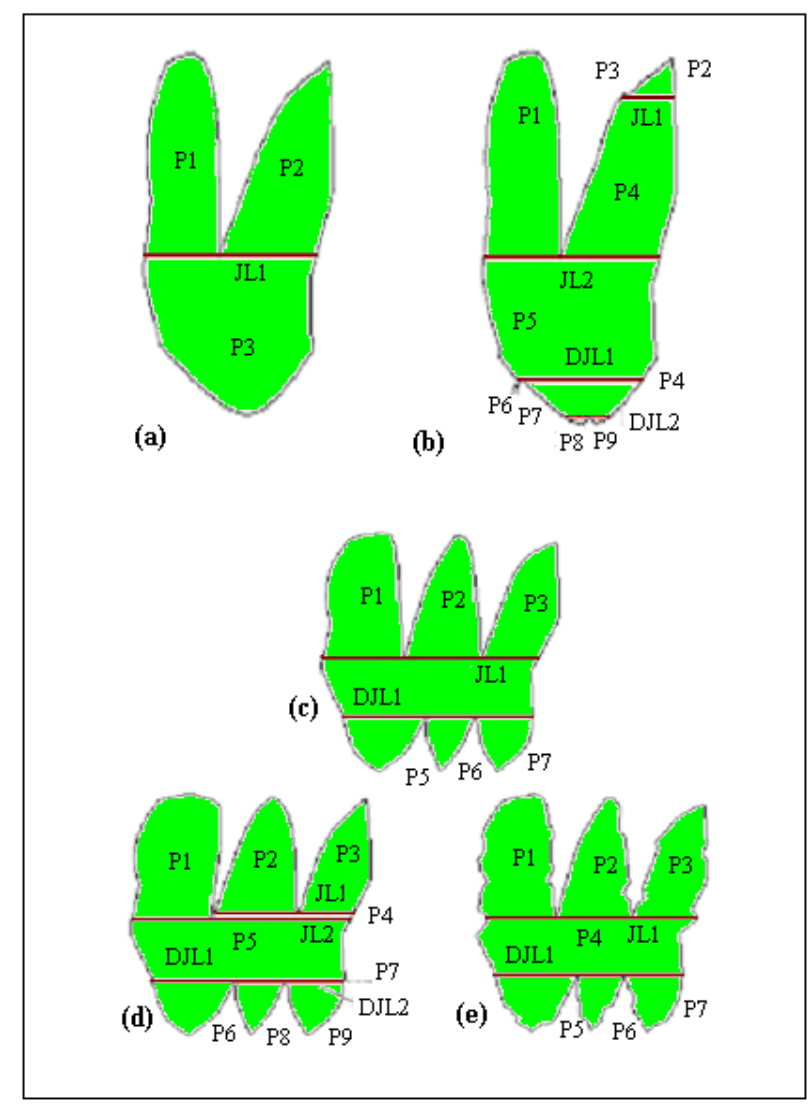

Fig. 2: Noisy outline shapes

\section{Matching descriptors of silhouettes}

\subsection{Matching Descriptors in Multi Scale Space}

As it is described above, XLWDOS descriptors are sensitive to noise. Therefore, it is necessary to take into account this noise in the matching process. In a previous work we have developed a method comparing silhouettes at different scales ${ }^{[20]}$ to eliminate noise from outline shape applying a convolution with a Gaussian filter. We obtain for each value of $\sigma$ a smoothed outline shape. We define the notion of multiscale XLWDOS descriptors as the set of descriptors computed using these smoothed outline shapes obtained at different scales (values of $\sigma$ ) from the initial outline shape. More the value of $\sigma$ increases, more the XLWDOS descriptor contains fewer elements whose number becomes steady from a certain value of $\sigma^{[20]}$. In this present work, we will see that it is possible to find same results with textual transformation of descriptors without Gaussian Smoothing.

\subsection{Reduction Method}

We define a noisy part, as a part whose length is 1,2 , 3 or $\mathbf{n}$ pixels. Each noisy part will be marked in the written descriptor using the two tags $\langle\mathbf{N}\rangle\langle/ \mathbf{N}\rangle$. For example, the XML structure of XLWDOS descriptors of silhouette 2.a and 2.b are:

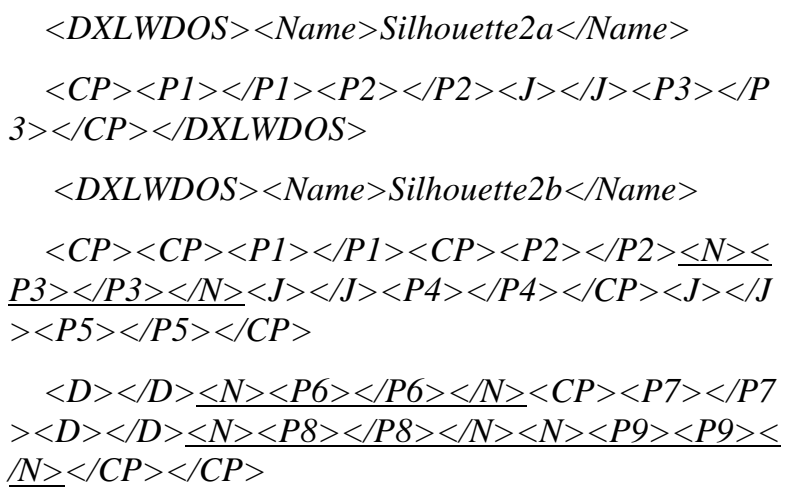

$</ D X L W D O S>$

These two descriptors cannot be matched because they have different structures. Therefore, the problem is to verify if we can reduce the two descriptors in order to maintain in their XML structures only their main parts. For this, we propose a reduction method that takes into account all possible positions of noisy parts in the XML structure.

Also, the XML structure of XLWDOS descriptors of silhouettes 2.c and 2.d are:

$$
\begin{aligned}
& <\text { DXLWDOS }><\text { Name }>\text { Silhouette2c }<\text { /Name }> \\
& <C P><C P><P 1></ P 1><P 2></ P 2><P 3></ P 3> \\
& <J></ J><P 4></ P 4></ C P> \\
& <D></ D><P 5></ P 5><P 6></ P 6><P 7></ P 7></ \\
& C> \\
& </ D X L W D O S> \\
& <\text { DXLWDOS }><\text { Name }>\text { Silhouette } 2 d</ \text { Name }> \\
& <C P><C P><P 1></ P 1><C P><P 2></ P 2><P 3> \\
& </> \\
& <J></ J><N><P 4></ P 4></ N></ C P> \\
& <J></ J><P 5></ P 5></ C P> \\
& <D></ D><P 6></ P 6><C P><\underline{N}><P 7></ P 7></ N>
\end{aligned}
$$




$$
\begin{aligned}
& <D></ D><P 8></ P 8><P 9></ P 9></ C P></ C P> \\
& </ D X L W D O S>
\end{aligned}
$$

The principle of our reduction method is as follows:

When the size of a part (Psi) is negligible in relation with sizes of main parts, the (Psi) will be considered as noisy and will be suppressed from the initial descriptor. The noisy part size is, therefore, less than a fixed threshold.

Let be:

$<C P>P s 1 \quad P s 2 \quad \ldots \quad$ Ps(i-1) Psi $P s(i+1) \ldots$ Psn $\quad J i$ $P m</ C P>$, a composed part according to XLWDOS Descriptor.

The same descriptor, after removing the noisy part (Psi), becomes:

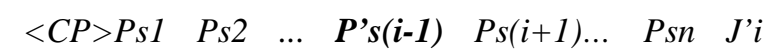
$P m</ C P>$.

P's(i-1) is generated from Ps(i-1) where the description of the left boundary of P's(i-1) is the same than the left boundary of $\operatorname{Ps}(\mathrm{i}-1)$. The right boundary of P's(i-1), is the right boundary of Ps(i-1) for which we add the noisy part (Psi) and the segments of separating lines adjacent to (Psi).

The separating line descriptor will also be modified according to the new part obtained after the reduction process.

Indeed all segments that are adjacent to (Psi), will be assigned to the new part P's(i-1).

The graphic illustration of this technique is given in Figure 3.

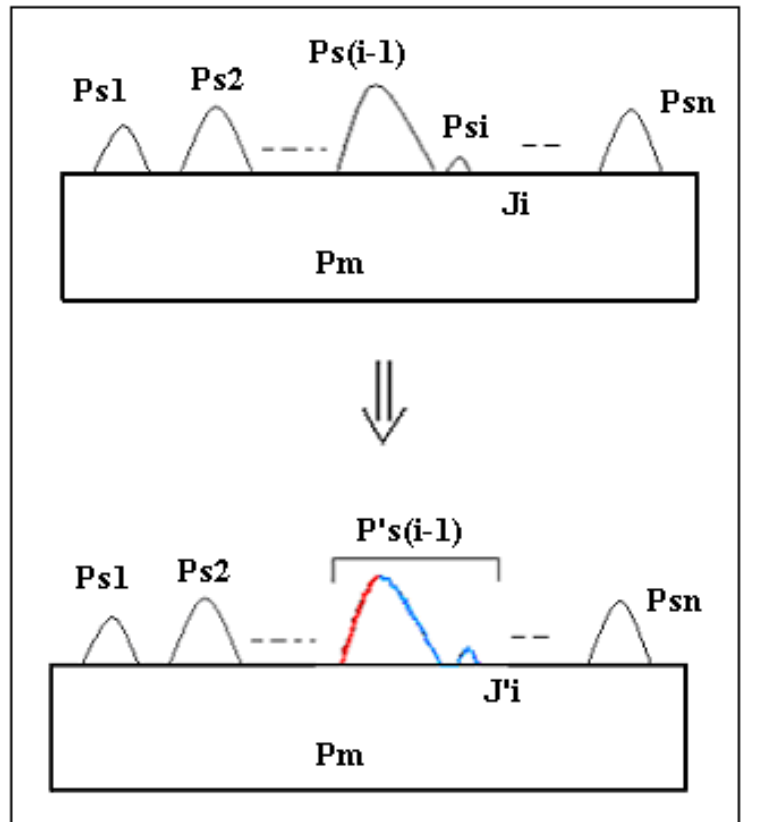

Fig. 3: Elimination of a noisy part in the textual description
If there is no part in the left of Psi (See Figure 4) then the contour of Psi becomes the continuation of the left boundary of part P's(i+1). Also the associated segments to part Psi in the line Ji will be attached to the new part P's(i+1).

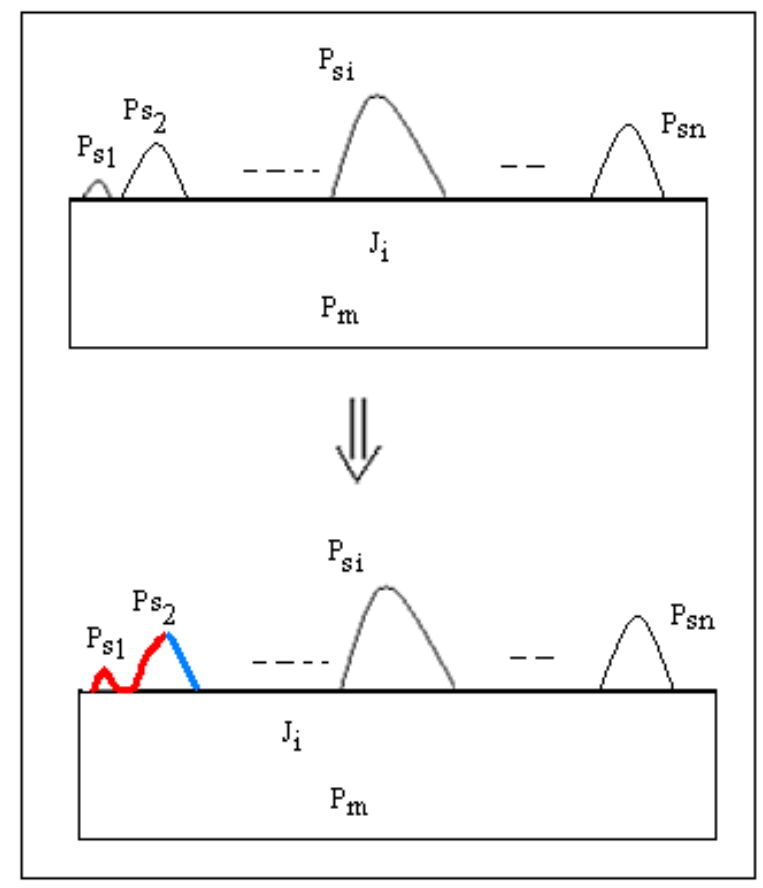

Fig. 4: Elimination of a noisy part in the left of the first main part in the descriptor

In case of all parts before the junction line are noisy, we will keep only one main part (the main part) after the reduction process as shown in Figure 5. The same process is applied if all parts after the disjunction line are noisy (are less than the fixed threshold)

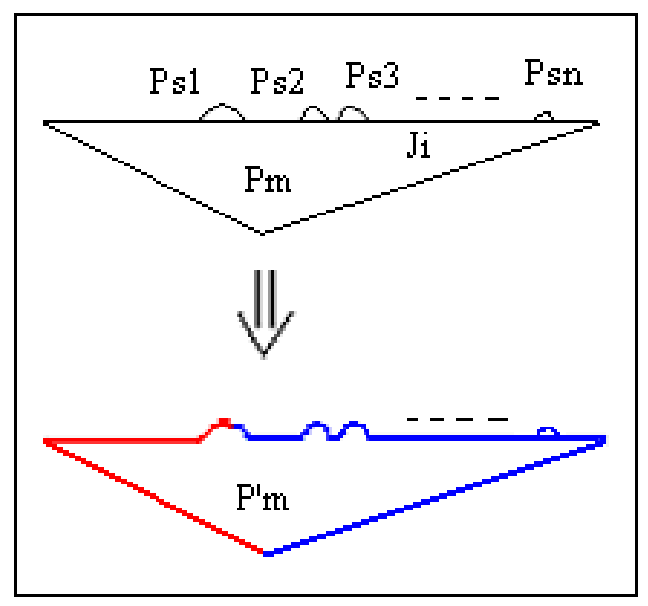

Fig. 5: Case of noisy parts before a junction line

This is a recursive process; it is applied to remove all noisy parts from the descriptor while parts sizes are less than the fixed threshold and while the two descriptors to be matched remain different during the reduction process. 


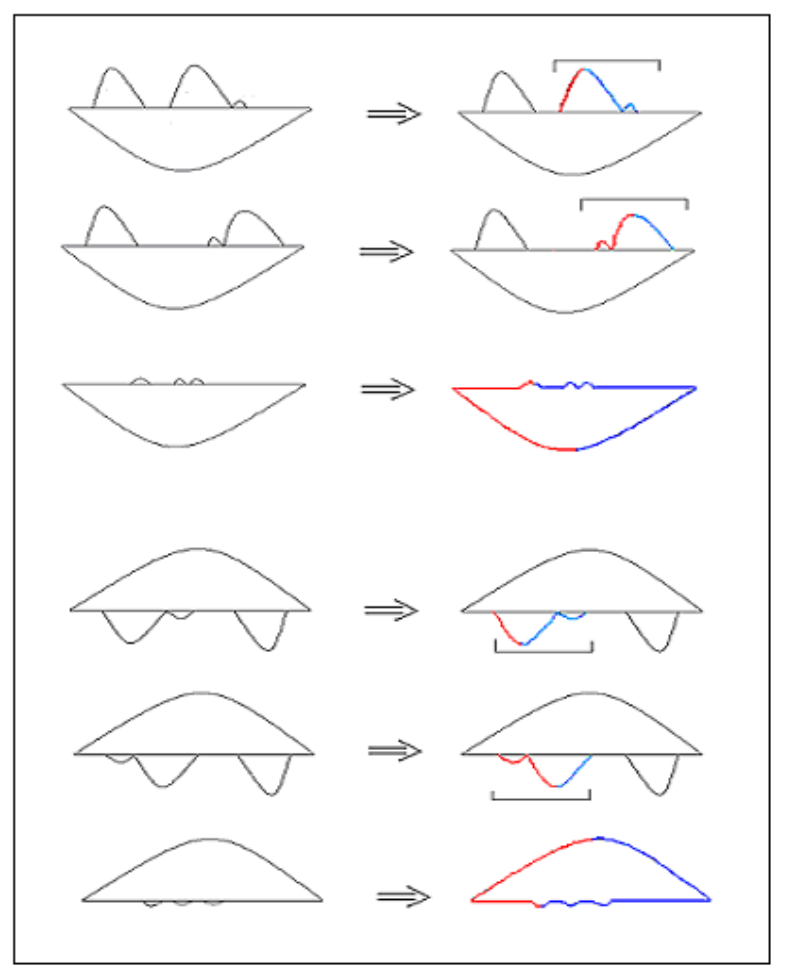

Fig. 6: Examples of descriptors reduction

For example, using the reduction method, the XML structure of XLWDOS descriptor of silhouette 2.d becomes:

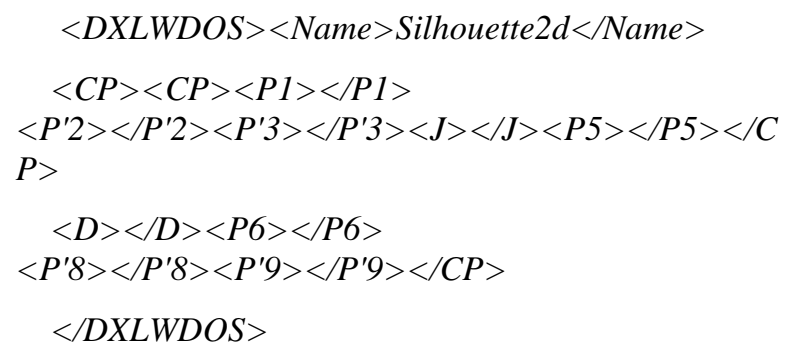

Now, the XML structure of both descriptors (Silhouette2c and Silhouette2d) are similar, therefore the matching of their elements may be done.

Other examples of transformations are given by graphic illustrations in Figure 6.

\section{Experimentation}

Different objects have been used to validate the proposed methods. We used many images taken in our laboratory (see Figure 7) and also some images of shapes built by B. Leibe and B. Schiele ${ }^{[21]}$ where each object is represented by 41 views spaced evenly over the upper viewing hemisphere (see Figure 8).

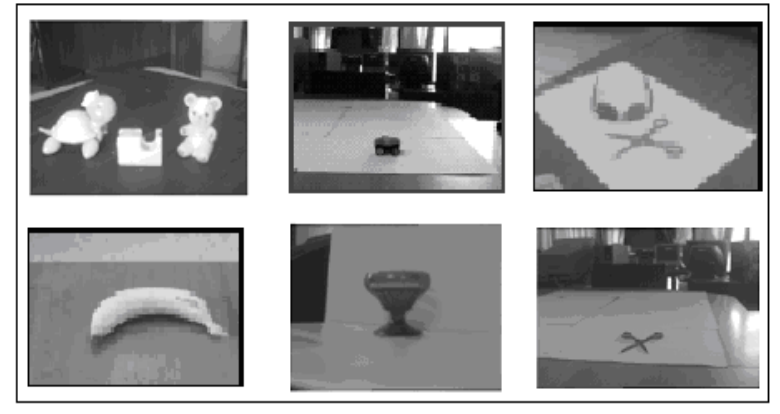

Fig. 7: Objects used in experimentation
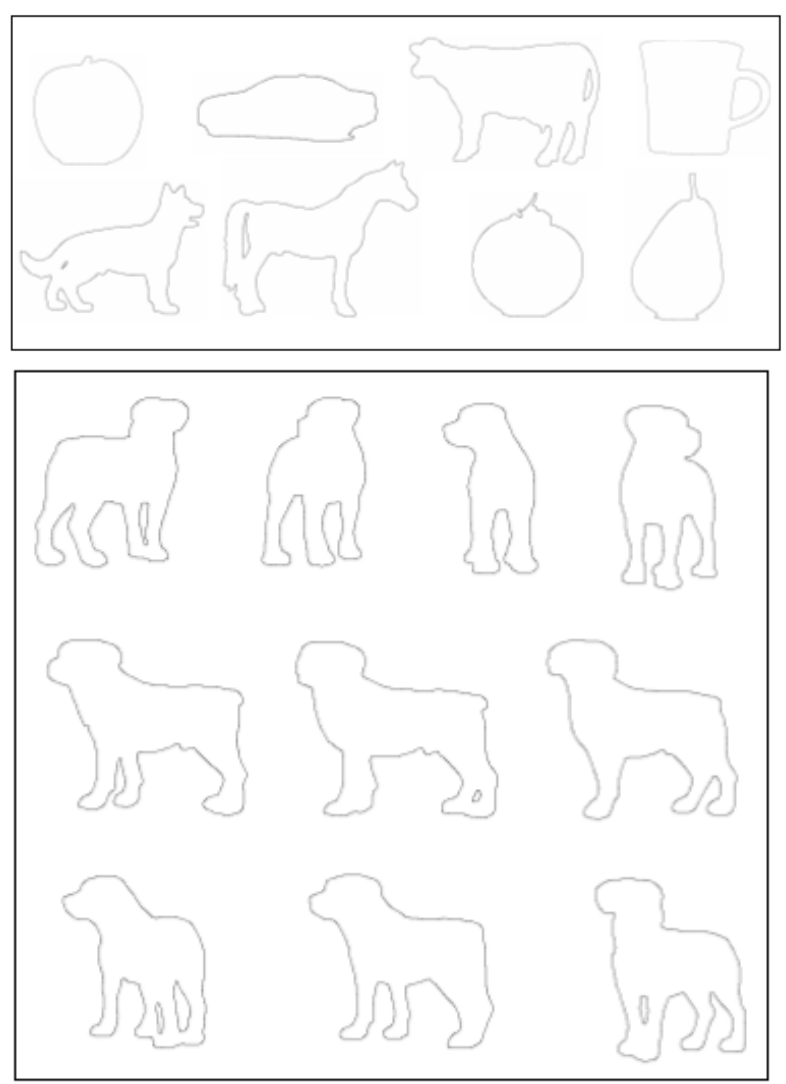

Fig. 8: A set of shapes of the database of Leibe and Schiele and some views of an object ${ }^{[21]}$

In Figure 9, we show two images of the same object (cup). There are parts and junction lines in the descriptor of the first image which could not be matched with other parts and lines in the descriptor of the second image.

After applying a convolution with a Gaussian filter for the two outline shapes, smoothed outlines shapes are obtained and the obtained XLWDOS descriptors of both two images become similar.

The matching problem has also been solved using our reduction method. The approach is been applied for the two noisy XLWDOS descriptors and gave same results. Indeed, the first noisy descriptor computed (of the first image) has the following structure: 


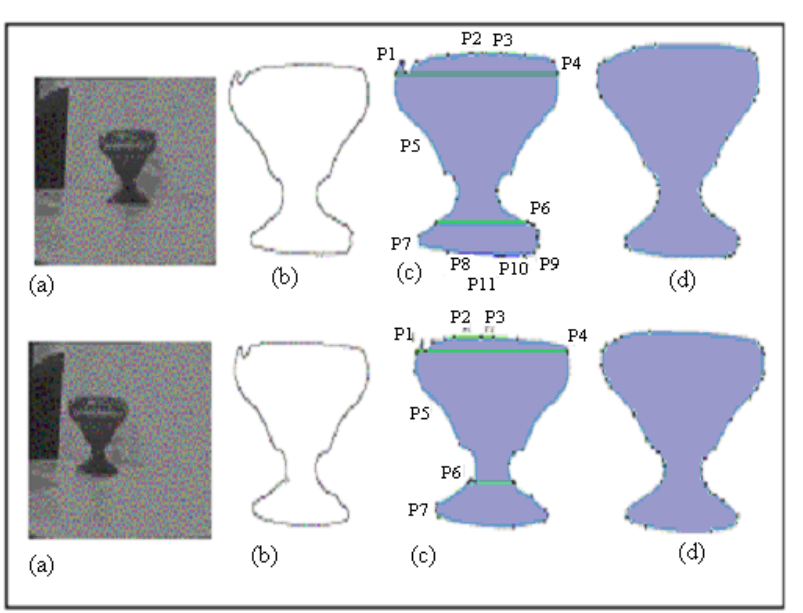

Fig. 9: For each image (a), the extracted outline shape of a cup (b), the result of applying XLWDOS description for outline shape (c) and the smoothed outline shape with $\sigma=10$ (d)

$$
<\text { DXLWDOS }><\text { Name }>\text { Cup } 1</ \text { Name }>
$$
$\underline{N>}$

$$
<C P><C P><C P><C P>\leq N><P 1></ P 1></ N><
$$

$$
\begin{aligned}
&><P 2></ P 2></ N><J></ J><P 3></ P 3></ C P><P 4 \\
&</ P 4><J></ J><P 5></ P 5> \\
&</ C P><N><P 6></ P 6></ N><J></ J><P 7></ P 7 \\
&></ C P> \\
&<D></ D><C P><N><P 8></ P 8></ N><D></ D> \\
&<N><P 10></ P 10></ N><N><P 11><P 11></ N> \\
&</ C P><N><P 9></ P 9></ N></ C P> \\
&</ D X L W D O S>
\end{aligned}
$$

This descriptor is reduced as follows:

$$
\begin{aligned}
& <\text { DXLWDOS }><\text { Name }>\text { Cup } 1</ \text { Name }> \\
& <C P><P 1 \cup P 2 \cup P 3></ P 1 \cup P 2 \cup P 3> \\
& <P 4></ P 4><J></ J><P 5 \cup P 6 \cup P 7 \cup P 8 \cup P 10 \cup P 11 \\
& \cup P 9></ P 5 \cup P 6 \cup P 7 \cup P 8 \cup P 10 \cup P 11 \cup P 9></ C P> \\
& </ D X L W D O S>
\end{aligned}
$$

where: $<\mathrm{P} 1 \cup \mathrm{P} 2 \cup \mathrm{P} 3></ \mathrm{P} 1 \cup \mathrm{P} 2 \cup \mathrm{P} 3>$ designates the part obtained as the union of the three parts $\mathrm{P} 1, \mathrm{P} 2$ and P3. Therefore the obtained descriptor may be written:

\section{$<$ DXLWDOS $><$ Name $>$ Cup $1<$ /Name $>$}

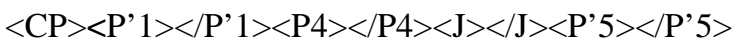
$</$ CP $></$ DXLWDOS $>$

The second noisy descriptor computed (of the second image) has the following structure:

$<$ DXLWDOS $><$ Name $>$ Cup $2<$ /Name $>$

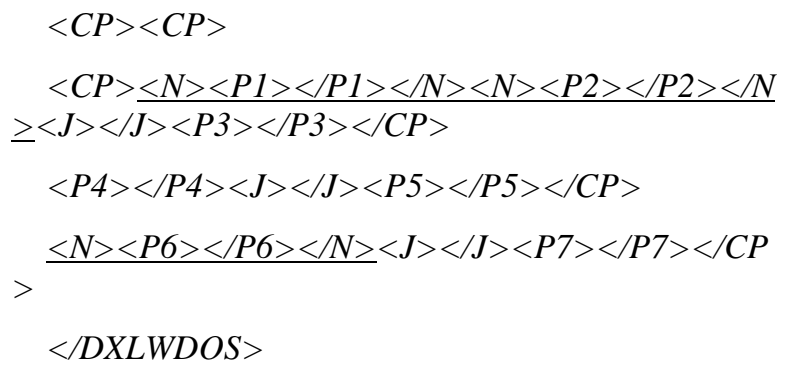

This descriptor is reduced as follows:

$<$ DXLWDOS $><$ Name $>$ Cup $2</$ Name $>$

$<C P><P 1 \cup P 2 \cup P 3></ P 1 \cup P 2 \cup P 3>$

$<P 4></ P 4><J></ J><P 5 \cup P 6 \cup P 7></ P 5 \cup P 6 \cup P 7$ $></ C P>$

$</ D X L W D O S>$

Therefore the obtained descriptor may be written:

$$
\begin{aligned}
& \quad<\text { DXLWDOS }><\text { Name }>\text { Cup } 2</ \text { Name }> \\
& <\text { CP }><P^{\prime \prime} 1></ P^{\prime \prime} 1><P 4></ P 4><J></ J><P \text {, } 5> \\
& </ P \text { ' } 5></ C P>
\end{aligned}
$$

$</ D X L W D O S>$

Now both descriptors (of cup1 and cup2) can be matched and their similarity is obtained by comparing the elementary contours of the obtained parts.

Two other examples of experiments are given in Figures 10 and 11, the same process is applied and similar results are obtained. Indeed, the descriptors of smoothed shapes become similar.

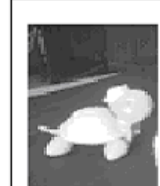

(a)

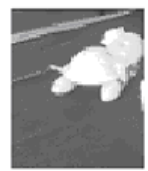

(a)

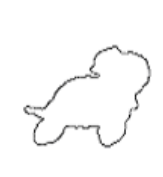

(b)

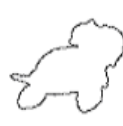

(b)

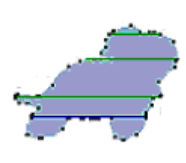

(c)

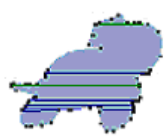

(c)

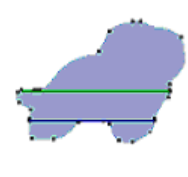

(d)

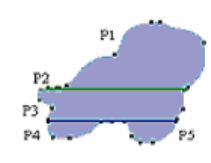

(d)
Fig. 10: Application of the reduction process on images of a turtle

For Figure 10, the two descriptors of the two objects to be matched are reduced to the following same descriptor:

$<\mathrm{CP}><\mathrm{CP}><\mathrm{P} 1 \cup \mathrm{P} 2></ \mathrm{P} 1 \cup \mathrm{P} 2><\mathrm{JL} 1></ \mathrm{JL} 1><\mathrm{P} 3><$ $/ \mathrm{P} 3></ \mathrm{CP}><\mathrm{P} 4></ \mathrm{P} 4><$ JL2 $></$ JL2 $>$ 
$<$ CP $><$ P5 $><$ /P5 $><$ DJL1 $><$ DJL1 $><$ P6 $\cup$ P7 $><$ /P6 $\cup$ P7 $>$ $</ \mathrm{CP}></ \mathrm{CP}>$

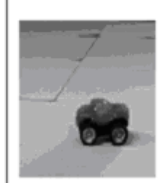

(a)

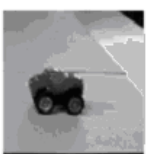

(a)

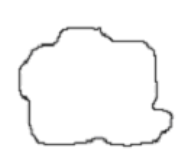

(b)

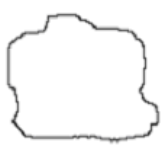

(b)

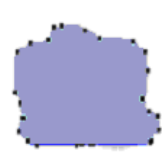

(c)

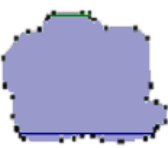

(c)

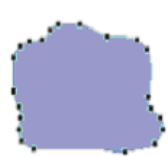

(d)

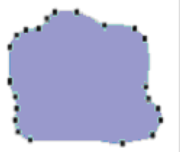

(d)
Fig. 11: Application of the reduction process on images of a turtle

In the same way, descriptors of silhouettes (a) and (b) in Figure 11 become:

$<$ CP $><\mathrm{P}^{\prime} 1></ \mathrm{P}^{\prime} 1><$ DJL'1 $><$ /DJL'1 $>$

$<\mathrm{P}^{\prime} 2 \cup \mathrm{P}^{\prime} 3></ \mathrm{P}^{\prime} 2 \cup \mathrm{P}^{\prime} 3></ \mathrm{CP}>$

Other experiments of pairs of images give following descriptors for silhouettes that look alike after application of the reduction process. Same descriptors are therefore obtained for the compared outline shapes.
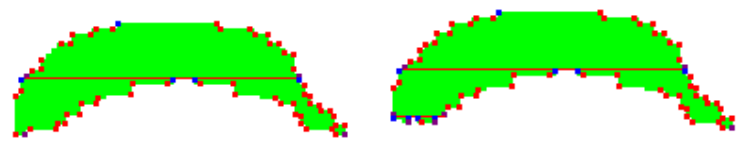

Fig. 12: Application of the reduction process on images of a banana

The obtained descriptors of silhouettes in Figure12 are:

$<\mathrm{CP}><\mathrm{P} 1><$ P $1><$ DJL1 $><$ DJL1 $><$ P2 $\cup \mathrm{P} 3></$ P $\cup \mathrm{P}$ $3></ \mathrm{CP}>$
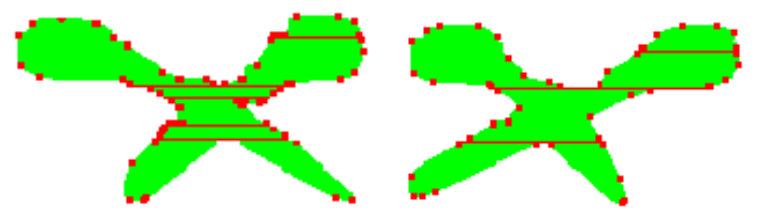

Fig. 13: Application of the reduction process on images of scissors

The obtained descriptors of silhouettes in Figure13 are:

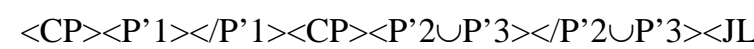
'1 $></$ JL' $1><\mathrm{P}^{\prime} 4></ \mathrm{P}^{\prime} 4>$

$</$ CP $><$ JL'2 $></$ JL'2 $><$ P'5 $^{\prime}<<$ /P'5 $><$ DJL'1 $><$ DJL'1 $>$

$<\mathrm{P}^{\prime} 6 \cup \mathrm{P}^{\prime} 7></ \mathrm{P}^{\prime} 6 \cup \mathrm{P}^{\prime} 7></ \mathrm{CP}>$
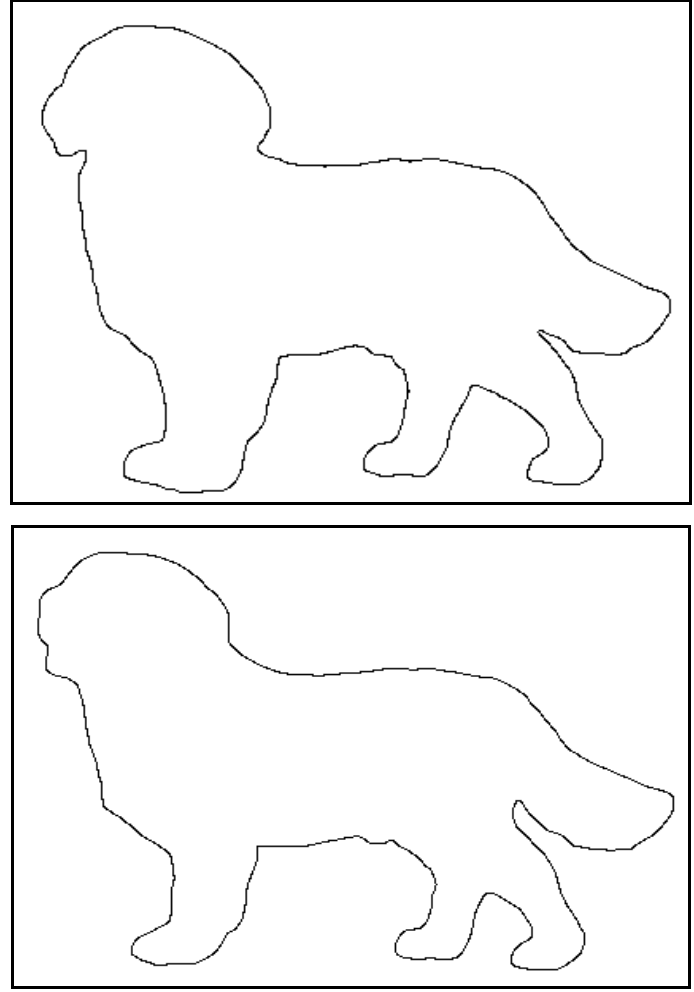

Fig. 14: Matching successive images of a dog ${ }^{\text {[21] }}$

The obtained descriptors of silhouettes in Figure14 (from the data base of Leibe and Schiele ${ }^{[21]}$ ) are:

$<\mathrm{CP}><\mathrm{CP}>\mathrm{P} 1<\mathrm{CP}>$ P2 $\mathrm{P} 3$ J1 $\mathrm{P} 4</ \mathrm{CP}>$ J2 $\mathrm{P} 5$ $</ \mathrm{CP}>$ D1 $<$ CP $>$ P6 D2 P8 $<$ CP $>$ P9 D3 P10 $<$ CP $>$ P11 D4 $<$ CP $>$ P12 D5 P14 P15 $</$ CP $>$ P13 $</$ CP $></$ CP $>$ $</ \mathrm{CP}>\mathrm{P} 7</ \mathrm{CP}>$

\section{Conclusion}

We proposed in this paper, an efficient method for silhouettes matching despite the presence of noise. The silhouettes are described according to the XLWDOS language. In order to permit a little difference in silhouettes shapes that generate unfortunately very different descriptors, a tolerance threshold is determined for XLWDOS descriptors by using thresholds for descriptors reducing and matching.

The proposed solution was illustrated by the writing of XLWDOS descriptors including the information of noisy parts. A reduction method has been developed in order to reduce their XML structures and let only main non-noisy parts.

Different positions of noisy parts were taken into account to achieve the matching process.

The conducted experiments show the usefulness of the proposed approach and demonstrate the possibility to use XLWDOS descriptors for real images applications. 


\section{References}

[1] Trinh, N.H., Kimia, B.B.: Skeleton Search: Category-Specific Object Recognition and Segmentation Using a Skeletal Shape Model. IJCV 94(2): 215-240, 2011.

[2] Sclaroff, S.: Deformable prototypes fpr encoding shapes categories in image databases, Pattern Recognition, 30(4), 1997.

[3] Mokhtarian, F., Mackworth, A. K.: A theory of multiscale, curvature-based shape representation for planar curves, IEEE PAMI, Vol 14, $\mathrm{N}^{\circ} 8$, August 1992.

[4] Mokhtarian, F.: Silhouette-Based isolated object recognition through curvature scale space, IEEE PAMI, Vol 17, N 5, 1995.

[5] Ma, T., Latecki, L.J.: From partial matching through local deformation to robust global shape similarity for object detection. CVPR: 1441-1448, 2011.

[6] Sethi, A., Renaudie, D., Kriegman, D., Ponce, J.: Curve and surface duals and the recognition of curved 3D objects from their silhouettes, I. J. C.V., 58 (1), 73-86, 2004.

[7] Wang, X., Bai, X., Liu, W., Latecki, L.J.: Feature context for image classification and object detection. CVPR 2011: 961-968, 2011.

[8] Orrite C., Herreo, J. E.: Shape matching of partially occluded curves invariant under projective transformation, Computer Vision and Image Understanding, vol. 93(1), 2004.

[9] Koenderink, J.J., Doorn, V.: the internal representation of solid shape with respect to vision, Biol. Cyber.,32, 1976.

[10] CYR, C. M., KIMIA, B.B.: A similarity-based aspect-graph approach to $3 \mathrm{D}$ object recognition, International Journal of Computer Vision, 57 (1), 5-22, 2004.

[11] Belongie, S., Malik, J., Puzicha, J.: Shape matching and object recognition using shape contexts, IEEE Transactions on P.A.M.I., Vol. 24, n 24, 2002.

[12] Ruberto, C. D.: Recognition of shapes by attributed skeletal graphs, Pattern Recognition, 37(1):21-31, 2004.

[13] Zhu, S.C., Yuille, A. L. : FORMS: A flexible object recognition and modeling system, Fifth International Conference on Computer Vision, June 20-23, M.I.T. Cambridge, 1995.

[14] Siddiqi, K., Kimia, B. B.: A shock grammar for recognition, Conference of Computer Vision and Pattern Recognition, 1996.
[15] Sebastian, T. B., Klein, P. N., Kimia, B. B.:Recognition of shapes by editing their shock graphs, IEEE Transactions on pattern Analysis and machine intelligence, Vol.26, n5, may 2004.

[16] Geiger, D., Liu, L., Kohn, R. V.: Representation and self-similarity of shapes, IEEE Transactions on pattern Analysis and machine intelligence, Vol.25, n¹, January 2003.

[17] Zhang, D., Lu, G.: Review of shape representation and description techniques, Pattern Recognition, 37(1):1-19, 2004.

[18] Campbell, R. J., Flynn, P. J.: A survey of freeform object representation and recognition techniques, Computer Vision and Image Understanding, 81, 166-210, 2001.

[19] Larabi, S., Bouagar, S., Trespaderne, F. M., Lopez, E. F.: LWDOS: Language for Writing Descriptors of Outline Shapes, In the LNCS proceeding of Scandinavian Conference on Image Analysis, June 29 - July 02, Gotborg, Sweden, 2003.

[20] Aouat, S., Larabi, S.: Matching Descriptors of Noisy Outline Shapes. Int. Journal of Image and Graphics. World Scientific Publisher. 2010.

[21] Leibe, B., Schiele, B.: Analyzing Appearance and Contour Based Methods for Object Categorization. In International Conference on Computer Vision and Pattern Recognition (CVPR'03), Madison, Wisconsin, June 2003.

\section{Authors’ Profiles}

Saliha AOUAT: Received the Ph.D. in Computer Science Department at USTHB University (Algiers) in 2009. She is currently Associate Professor at the Computer Science Department of the University of Sciences and Technologies, Algiers, Algeria. She is an author of numerous publications for conferences, proceedings, and journals. Her research interests include computer vision, Image and Texture Analysis, 3D Vision, Content based image indexing and retrieval, and pattern recognition.

Slimane LARABI: Received the Ph.D. in Computer Science from the National Institute Polytechnic of Toulouse, France, 1991. In January 1992, he joined the computer Science Department of the University of Science and Technology Houari Boumediene, Algiers, Algeria where he is currently Professor and leads research in Computer Vision Group of the Laboratory of Artificial Intelligence Research.

His work spans a range of topics in vision including Image description, Human action recognition, Head and Body pose estimation, Video Analysis, Knowledge modeling and representation for computer vision. He has written several research papers and supervised several $\mathrm{PhD}$. He has also conducted several national research projects. 